\title{
ON THE DJL CONJECTURE FOR ORDER 6
}

\author{
NAOMI SHAKED-MONDERER
}

Abstract. In 1994 Drew, Johnson and Loewy conjectured that for $n \geqslant 4$, the cp-rank of any $n \times n$ completely positive matrices is at most $\left\lfloor n^{2} / 4\right\rfloor$. Recently this conjecture has been proved for $n=5$ and disproved for $n \geqslant 7$, leaving the case $n=6$ open. We make a step toward proving the conjecture for $n=6$. We show that if $A$ is a $6 \times 6$ completely positive matrix that is orthogonal to an exceptional extremal copositive matrix, then the cp-rank of $A$ is at most 9 .

Mathematics subject classification (2010): 15B48, 15 A23.

Keywords and phrases: Completely positive matrix, cp-rank, copositive matrix, exceptional matrix, minimal zeros.

\section{REFERENCES}

[1] Francesco Barioli And Abraham Berman, The maximal cp-rank of rank $k$ completely positive matrices, Linear Algebra Appl., 363 (2003): 17-33.

[2] A. BERMAN, N. SHAKED-MONDERER, Remarks on completely positive matrices, Linear and Multilinear Algebra, 44 (1998): 149-163.

[3] A. Berman, N. Shaked-Monderer, Completely Positive Matrices, World Scientific (2003).

[4] I. BOMZE, W. SCHACHINGER AND R. ULLRICH, From seven to eleven: completely positive matrices with high cp-rank, Linear Algebra Appl., 459 (2014): 208-221.

[5] I. BOMZE, W. SCHACHINGER AND R. UlLRICH, New lower bound and asymptotics for the cp-rank, SIAM J. Matrix Anal. Appl. 36 (2015), 20-37.

[6] P. H. DiAnanda, On non-negative forms in real variables some or all of which are non-negative, Proc. Cambridge Philos. Soc. 58 (1962), 17-25.

[7] P. J. C. Dickinson, An improved characterization of the interior of the completely positive cone, Electron. J. Linear Algebra 20 (2010), 723-729.

[8] P. J. C. Dickinson, M. DÜR, L. GiJben And R. Hildebrand, Irreducible elements of the copositive cone, Linear Algebra and its Applications 439 (2013), 1605-1626.

[9] R. Diestel, Graph Theory, fourth edition, Springer-Verlag, Heidelberg (2010).

[10] J. H. Drew, C. R. Johnson, R. LoEWy, Completely positive matrices associated with $M$-matrices, Linear Multilinear Algebra, 37 (1994): 303-310.

[11] J. HANNAH, T. J. LAFFEY, Nonnegative factorization of completely positive matrices Linear Algebra Appl., 55 (1983): 1-9.

[12] R. Hildebrand, The extremal rays of the $5 \times 5$ copositive cone, Linear Algebra Appl., 437 (2012): 1538-1547.

[13] R. Hildebrand, Minimal zeros of copositive matrices, Linear Algebra Appl., 459 (2014): $154-174$.

[14] A. J. Hoffman And F. Pereira, On copositive matrices with -1, 0, 1 entries, J. Combin. Theory Ser. A 14 (1973), 302-309.

[15] R. LOEWY AND B.-S. TAM, CP rank of completely positive matrices of order 5, Linear Algebra Appl., 363 (2003): 161-176.

[16] B. Shader, N. Shaked-Monderer, D. B. Szyld, Nearly positive matrices, Linear Algebra Appl., 449 (2014): 520-544.

[17] N. SHAKED-MONDERER, Bounding the cp-rank by graph parameters, Electron. J. Linear Algebra, 28 (2015): 99-116. 
[18] N. Shaked-Monderer, I. M. Bomze, F. Jarre, And W. Schachinger, On the cp-rank and minimal cp factorizations of a completely positive matrix, SIAM J. Matrix Anal. Appl., 34 (2013): $355-368$.

[19] N. Shaked-Monderer, A. Berman, I. M. Bomze, F. Jarre, and W. Schachinger, New results on co(mpletely) positive matrices, Linear Multilinear Algebra, 63 (2015): 384-396.

[20] N. Shaked-Monderer, A. Berman, M. DÜr And M. Rajesh Kannan, SPN completable graphs, Linear Algebra Appl., 509 (2016): 82-113. 